\title{
Waste Utilization of Synthetic Carbon Quantum Dots Based on Tea and Peanut Shell
}

\author{
Jing Zhu, ${ }^{1}$ Fengyuan Zhu, ${ }^{2}$ Xiaona Yue, ${ }^{1}$ Peirong Chen, ${ }^{1}$ Yue Sun, ${ }^{1}$ Liang Zhang, \\ Dongdong $\mathrm{Mu}\left(\mathbb{D},{ }^{1,3}\right.$ and Fei $\mathrm{Ke} \mathbb{i D}^{1}$ \\ ${ }^{1}$ Department of Applied Chemistry \& State Key Laboratory of Tea Plant Biology and Utilization, Anhui Agricultural University, \\ Hefei, 230036 Anhui, China \\ ${ }^{2}$ Key Laboratory of Organic Synthesis of Jiangsu Province, College of Chemistry, Chemical Engineering and Materials Science \& \\ Collaborative Innovation Center of Suzhou Nano Science and Technology, Soochow University, Suzhou, 215123 Jiangsu, China \\ ${ }^{3}$ School of Food Science and Engineering, Key Laboratory for Agricultural Products Processing of Anhui Province, Hefei University \\ of Technology, Hefei 230009, China
}

Correspondence should be addressed to Dongdong Mu; d.mu@hfut.edu.cn and Fei Ke; kefei@ahau.edu.cn

Received 28 February 2019; Revised 27 April 2019; Accepted 30 May 2019; Published 8 July 2019

Guest Editor: Mingheng Li

Copyright ( 2019 Jing Zhu et al. This is an open access article distributed under the Creative Commons Attribution License, which permits unrestricted use, distribution, and reproduction in any medium, provided the original work is properly cited.

According to the concept of waste utilization and environmental protection, carbon quantum dots (CQDs) with high quantum yield and good stability were synthesized from waste tea leaves and peanut shells by a one-step hydrothermal method. In this study, we explored the synthetic conditions, structures, and optical properties of CQDs. Their unique characteristics of emitting strong and steady blue fluorescence under excitation of ultraviolet light and possessing of plenty of hydrophilic groups on a surface conferred CQD potentials in the field of biomarkers and analytical detection.

\section{Introduction}

Carbon quantum dots (CQDs) are quasizero-dimensional carbon nanoparticles with an average particle size of less than $10 \mathrm{~nm}$, and the surfaces of CQDs contain many organic functional groups. The synthesis of the CQD process is simple, and the raw materials are low-cost. Due to their low toxicity, good biocompatibility, high chemical stability, and good light stability, CQDs have potential applications in many fields such as biomarkers [1], ion detection, and photocatalysis [2-6]. Meanwhile, a CQD preparation method has many advantages, such as abundant carbon sources, simple reaction equipment, and high fluorescent efficiency. The synthetic CQDs are soluble in water. $0.2 \mathrm{~mL}$ tea soup and $0.1 \mathrm{~mL}$ CQD solution were added into the tube, and the solution was mixed [7-11]. To date, there are many ways to synthesize carbon quantum dots: for example, electrochemistry, combustion, ultrasonic, pyrolysis, and microwave. But most of the methods are complex, costly, and toxic. The toxic substances used in the experiment have always been the problem that researchers are eager to solve. Therefore, it is very important for us to explore a simple, low-cost, and low-toxic synthesis method. [12] In addition to the reagents purified in laboratory, household waste which provides a rich carbon source can also be used as raw materials to synthesize CQDs [13-17]. In countries where people like drinking tea, such as East Asia and UK, large amounts of discarded tea leaves are produced every year. The same neglected situation also occurred on the peanut shell. The number of peanut shells discarded each year is also very large, and the shell is difficult to recycle. The reuse of wastes has become one of the hottest scientific research topics nowadays. In this study, a hydrothermal synthesis method for preparing CQDs from abandoned tea leaves and peanuts shell was created (Figure 1), by which the generated CQDs become soluble in water properly and gain excellent fluorescence characteristics and good stability. 


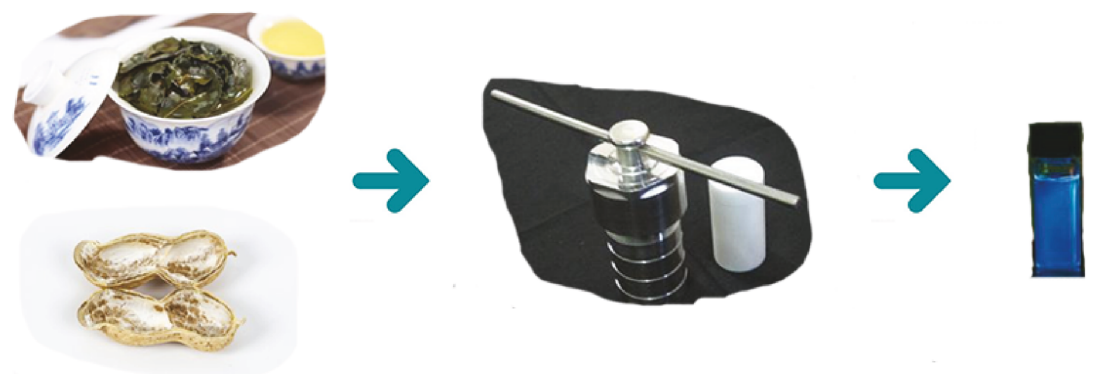

FIgURe 1: Process routing of CQDs.

\section{Experimental Section}

2.1. Apparatus and Reagents. Ethanol and acetic acid were purchased from Sinopharm Chemical Reagent Co. Ltd. All reagents used were of analytical grade without further treatment. The water used throughout was ultrapure water. Dianhong black tea samples were collected from Yunnan Dianhong Black Tea Group Co. Ltd. Abandoned Tieguanyin Tea (purchased on Taobao) was soaked in hot water at $100^{\circ} \mathrm{C}$ for 5 times and dried in an oven to get the discarded tea. Peanut shell was washed with pure water and dried in an oven at $100^{\circ} \mathrm{C}$, then grinded into powder by a crusher, packed in a sealed bag, and kept at $4^{\circ} \mathrm{C}$.

A Cary Eclipse fluorescence spectrophotometer (Agilent Technologies, America) and a UV-2100 ultraviolet spectrophotometer (Shimadzu Corporation, Japan) were used to record fluorescence spectra and absorption spectra of CQDs, respectively. A HT-7700 transmission electron microscope (TEM) was used to observe the size and morphology.

2.2. Synthesis of Tea CQDs. The preparation process of CQDs is shown in Figure 1. Firstly, ground tea powder was dissolved in $30 \mathrm{~mL} 3 \%$ acetic acid solution and sonicated for $15 \mathrm{~min}$. Then, the mixture was poured into a $50 \mathrm{~mL}$ PPL reactor to react at $200^{\circ} \mathrm{C}$ for $4 \mathrm{~h}$ and cooled down to room temperature after the reaction finished. The obtained yellow liquid was filtered twice by a $0.22 \mu \mathrm{m}$ microfiltration membrane and dialyzed in a dialysis bag for $24 \mathrm{~h}$ to obtain CQD solution; the pure water was changed every 6 hours. A light yellow CQD powder was obtained after vacuum drying.

2.3. Synthesis of Peanut Shell CQDs. Firstly, peanut shell powder was dissolved in $30 \mathrm{~mL} \mathrm{3 \%}$ acetic acid solution and sonicated for $15 \mathrm{~min}$. Then, the mixture was poured into the $50 \mathrm{~mL}$ PPL reactor to react at $200^{\circ} \mathrm{C}$ for $4 \mathrm{~h}$ and cooled down to room temperature after the reaction finished. The obtained yellow liquid was filtered twice by a $0.22 \mu \mathrm{m}$ microfiltration membrane and dialyzed in a dialysis bag for $24 \mathrm{~h}$ to obtain a CQD solution.

2.4. Characterization of CQDs. The luminescence properties of samples were determined by a fluorescence spectrophotometer and an ultraviolet spectrophotometer. The morphology and size of the samples were observed and determined using a TEM.

2.5. Application of Quantum Dots in Detection. The purchased black tea had been divided into 8 experimental grades
(Super, First, Second, Third, Fourth, Fifth, Sixth, and Outside) based on its quality. Three samples ( $0.1 \mathrm{~g}$ per sample) were taken from each tea grade, yielding a total of 24 samples. $100 \mathrm{mg}$ of each tea samples was soaked in water at $90^{\circ} \mathrm{C}$ for $3 \mathrm{~min}$. After adding $3 \mathrm{~mL}$ of water to a $5 \mathrm{~mL}$ centrifuge tube, $0.2 \mathrm{~mL}$ of tea soup and $0.1 \mathrm{~mL}$ of quantum dot solution were added and the mixture was shaken. The sample was subjected to fluorescence detection, and the data was saved for analysis.

\section{Results and Discussion}

3.1. The Effect of Carbon Source Dosage on CQD Preparation. When using tea as a carbon source, we weighed $0.25 \mathrm{~g}, 0.50 \mathrm{~g}$, $0.75 \mathrm{~g}, 1.00 \mathrm{~g}, 1.25 \mathrm{~g}$, and $1.50 \mathrm{~g}$ of tea to get reactions separately. Through the fluorescence spectrum (Figure 2(a)), the fluorescence intensity did not change anymore when the amount of the tea reaches $1 \mathrm{~g}$. It showed that the optimal reaction of tea CQDs is $1 \mathrm{~g}$, and the best ratio of waste Tieguanyin tea powder quality to $3 \%$ acetic acid solution is $0.033 \mathrm{~g} / \mathrm{mL}$. As shown in Figure 2(b), $0.25 \mathrm{~g}, 0.50 \mathrm{~g}, 0.75 \mathrm{~g}, 1.00 \mathrm{~g}, 1.25 \mathrm{~g}$, $1.50 \mathrm{~g}, 1.75 \mathrm{~g}, 2.00 \mathrm{~g}$, and $2.25 \mathrm{~g}$ of peanut shell were used to get reactions separately. Different from tea, when the amount of peanut shell exceeds $1.5 \mathrm{~g}$, the fluorescence intensity showed a significant decrease indicating the optimum amount of the peanut shell powder for preparing the CQDs is $1.5 \mathrm{~g}$.

3.2. The Effect of Reaction Time on CQD Preparation. For tea CQDs, we recorded the fluorescence spectrum (Figure 3(a)) of the reaction time in $120,180,240,300,360,420$, and $480 \mathrm{~min}$, respectively. The results showed the fluorescence intensity did not change after the reaction time reached $300 \mathrm{~min}$, which meant the optimal reaction time of tea carbon quantum dots was $300 \mathrm{~min}$. The fluorescence intensity of the peanut shell is shown in Figure 3(b), the measured fluorescence intensity was the strongest when the reaction time was $300 \mathrm{~min}$. After that, as the reaction time increased, the fluorescence intensity gradually decreased. It showed that the optimal reaction time of peanut shell CQDs was $300 \mathrm{~min}$.

3.3. The Effect of Reaction Temperature on CQD Preparation. The tea reaction solution was reacted at $100^{\circ} \mathrm{C}, 150^{\circ} \mathrm{C}, 180^{\circ} \mathrm{C}$, $200^{\circ} \mathrm{C}, 210^{\circ} \mathrm{C}, 220^{\circ} \mathrm{C}, 230^{\circ} \mathrm{C}, 240^{\circ} \mathrm{C}, 250^{\circ} \mathrm{C}, 260^{\circ} \mathrm{C}$, and $270^{\circ} \mathrm{C}$, for $300 \mathrm{~min}$ respectively. As shown in Figure 4(a), as the temperature raised, fluorescence intensity first increased, then decreased, and the intensity reached a maximum at $250^{\circ} \mathrm{C}$. Similarly, we also optimized the reaction temperature of peanut shell CQDs. The tests were carried out at $100^{\circ} \mathrm{C}, 150^{\circ} \mathrm{C}$, 


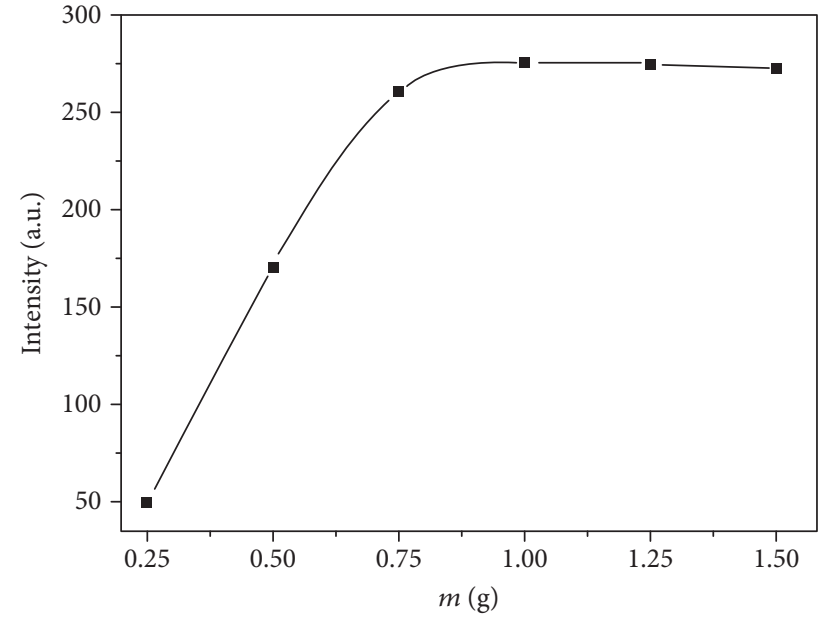

(a)

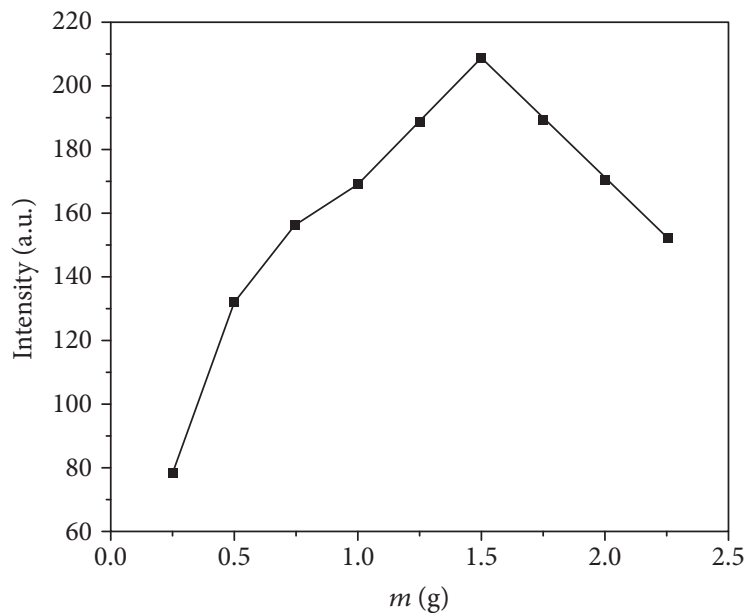

(b)

FIgURE 2: Influence of carbon source dosage of (a) tea and (b) peanut shell.

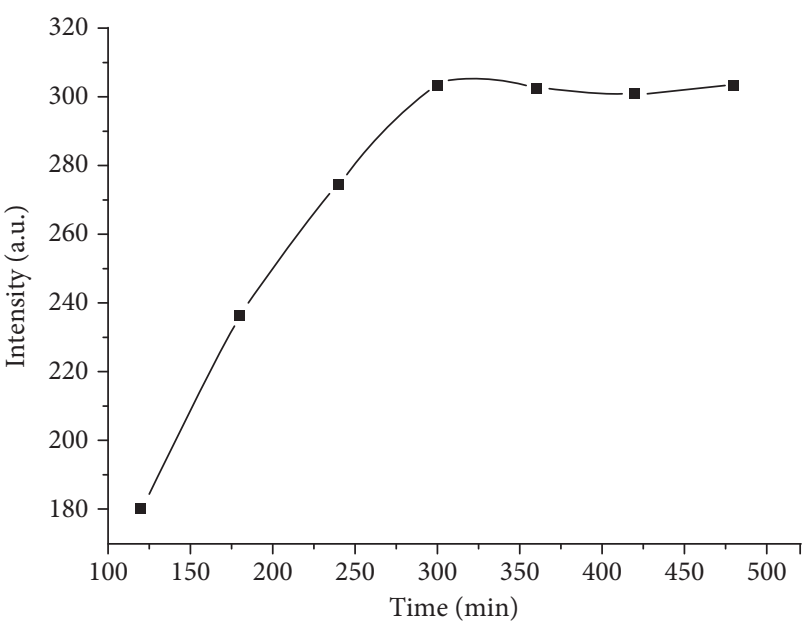

(a)

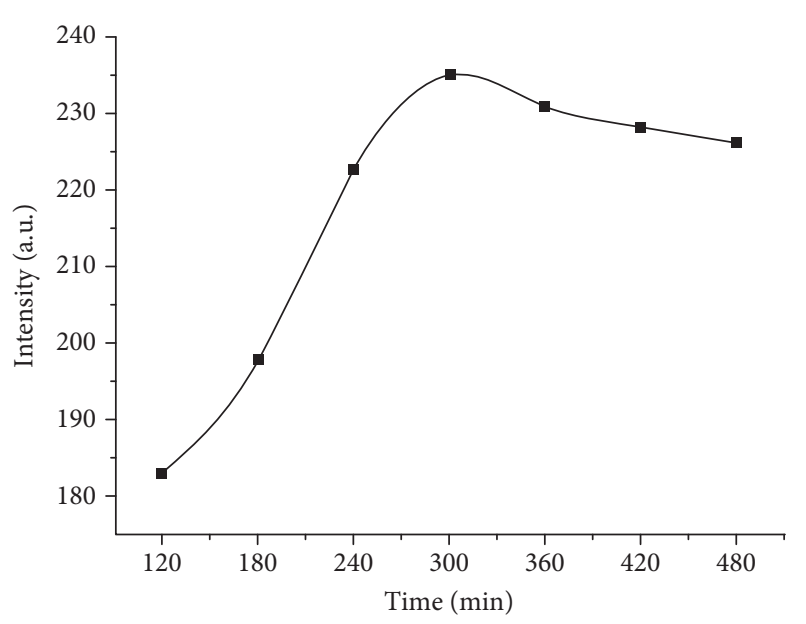

(b)

FIgURE 3: Influence of reaction time of (a) tea and (b) peanut shell.

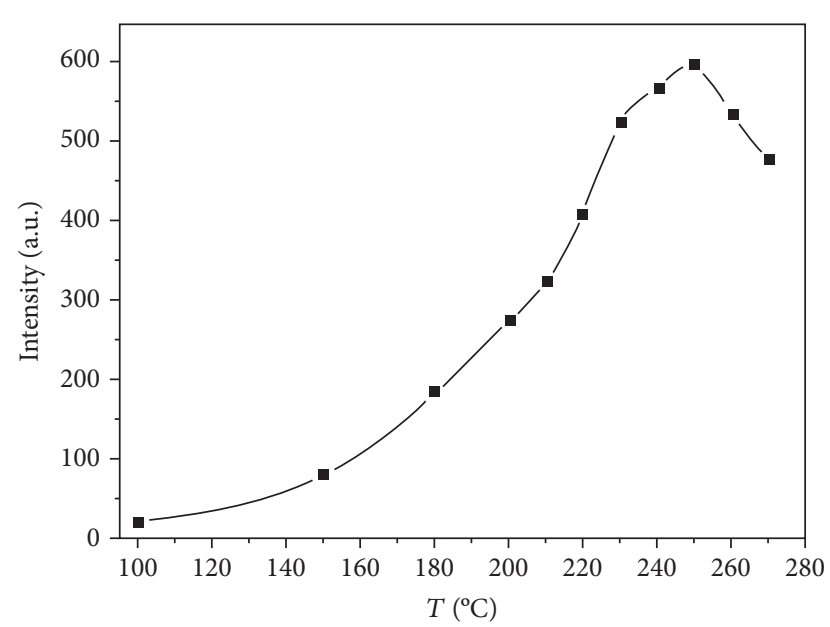

(a)

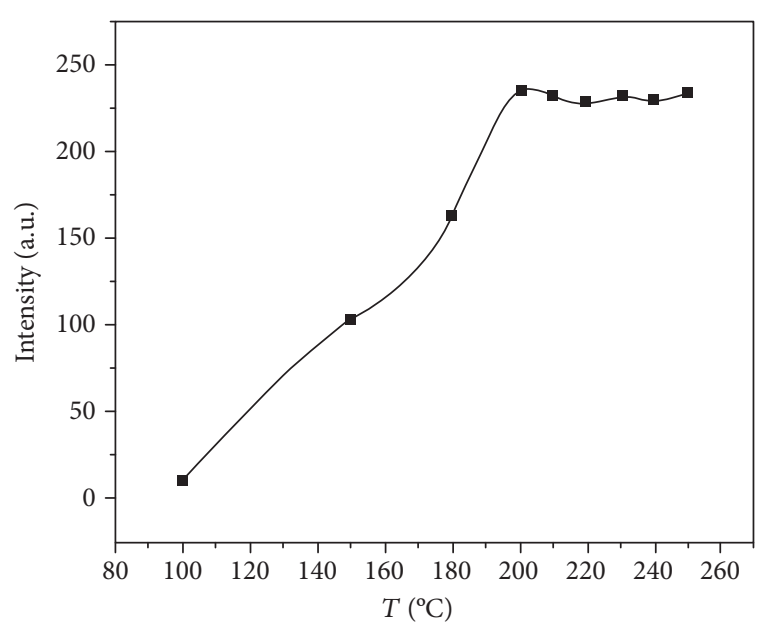

(b)

FIGURE 4: Influence of reaction temperature of (a) tea and (b) peanut shell. 


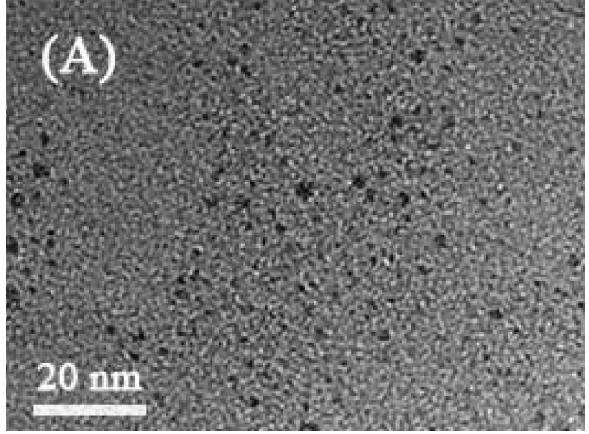

(a)

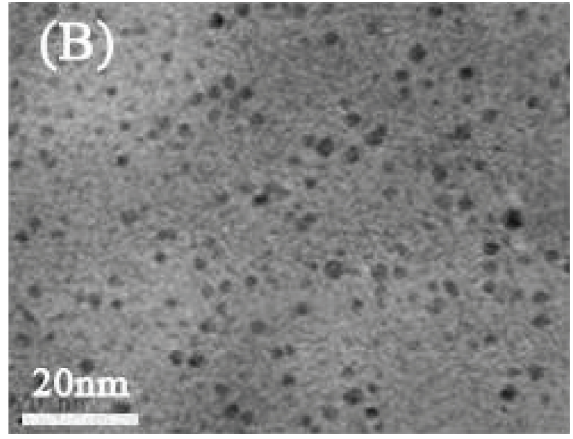

(b)

FIgURE 5: Transmission electron microscopy micrograph of CQDs of (a) tea and (b) peanut shell.

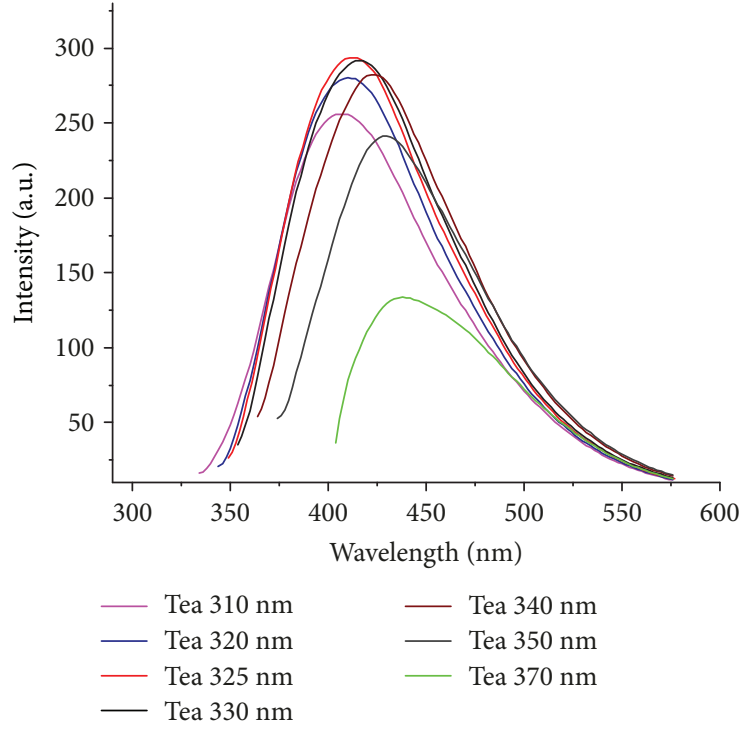

(a)

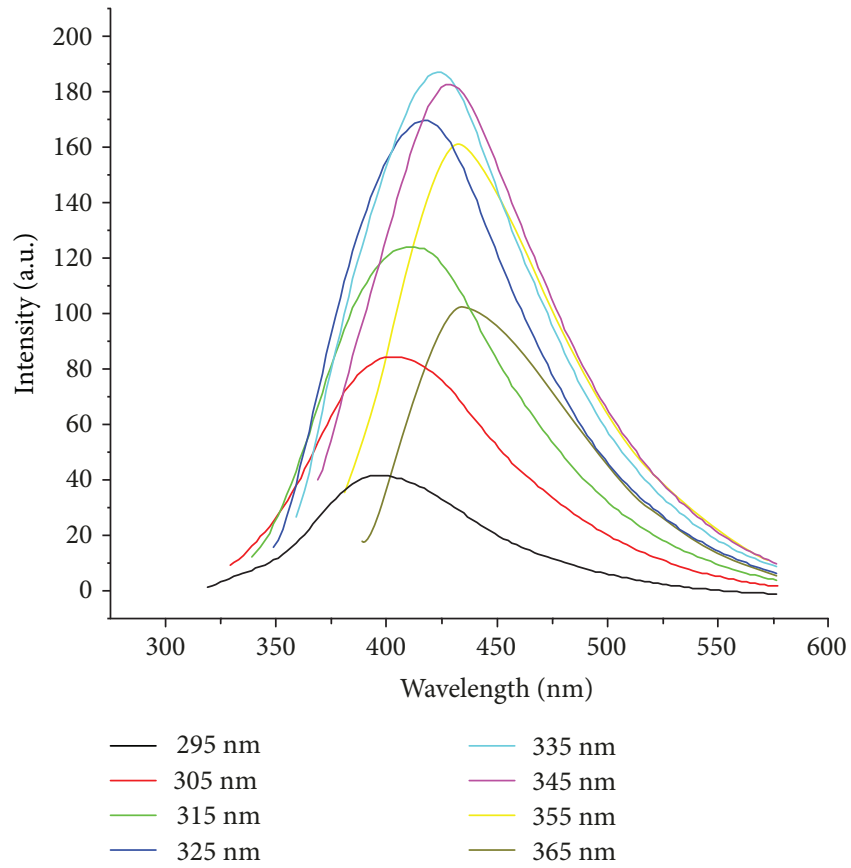

(b)

FIGURE 6: Fluorescence spectrum of CQDs of (a) tea and (b) peanut shell.

$180^{\circ} \mathrm{C}, 200^{\circ} \mathrm{C}, 210^{\circ} \mathrm{C}, 220^{\circ} \mathrm{C}, 230^{\circ} \mathrm{C}, 240^{\circ} \mathrm{C}$, and $250^{\circ} \mathrm{C}$. From Figure $4(\mathrm{~b})$, when the holding temperature exceeds $200^{\circ} \mathrm{C}$, the measured fluorescence intensity did not change significantly. It showed that the optimal preparation temperature for carbon quantum dots is $200^{\circ} \mathrm{C}$.

3.4. Spectral Properties of CQDs. According to the TEM test results (Figure 5), there was no obvious difference in the morphology between tea CQDs and peanut shell CQDs. The diameter was 7-9 nm, its shape was spherical, and the dispersion was uniform, which indicated the tea CQDs and peanut shell CQDs were more stable in water.

The tea CQDs had the strongest fluorescence intensity at $325 \mathrm{~nm}$ under different excitation light conditions and emitted blue light fluorescence.

Therefore, the wavelength of the best excitation light was $325 \mathrm{~nm}$; as the wavelength of the excitation light increases, the fluorescence appeared red-shifted. It is shown in Figure 6 that optimum excitation wavelength of peanut shell CQDs was $335 \mathrm{~nm}$ and the luminescence was also concentrated in the blue light region.

Through the infrared spectrum (Figure 7), it could be seen that there were some differences in the functional groups between the two carbon quantum dots. Quantum dots using tea as a carbon source had more functional groups on the surface than the others, such as $-\mathrm{COOH}$ and $-\mathrm{OH}$, so the water solubility and stability of CDQs were very good.

3.5. Application of CQDs. As we knew, many substances could cause the quenching of quantum dots. The content of tea was very rich, and tea soup could cause the quenching of CQDs. There were differences in the ingredients contained in different grades of tea. However, such a difference was relatively subtle and had high requirements for the accuracy of the test instrument. This high sensitivity of carbon quantum could be used to identify such subtle differences. We took 


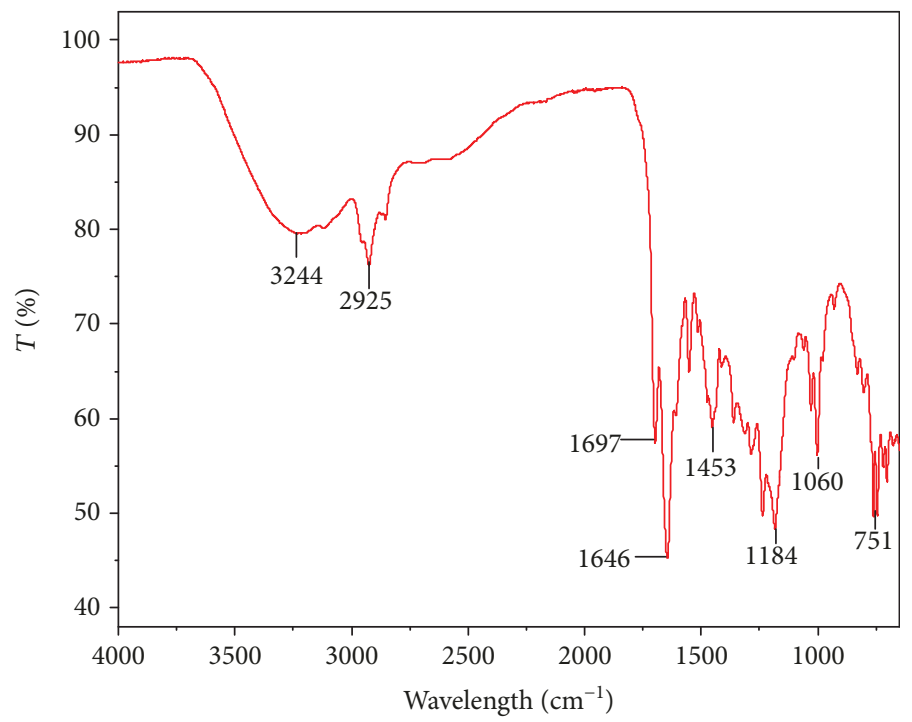

(a)

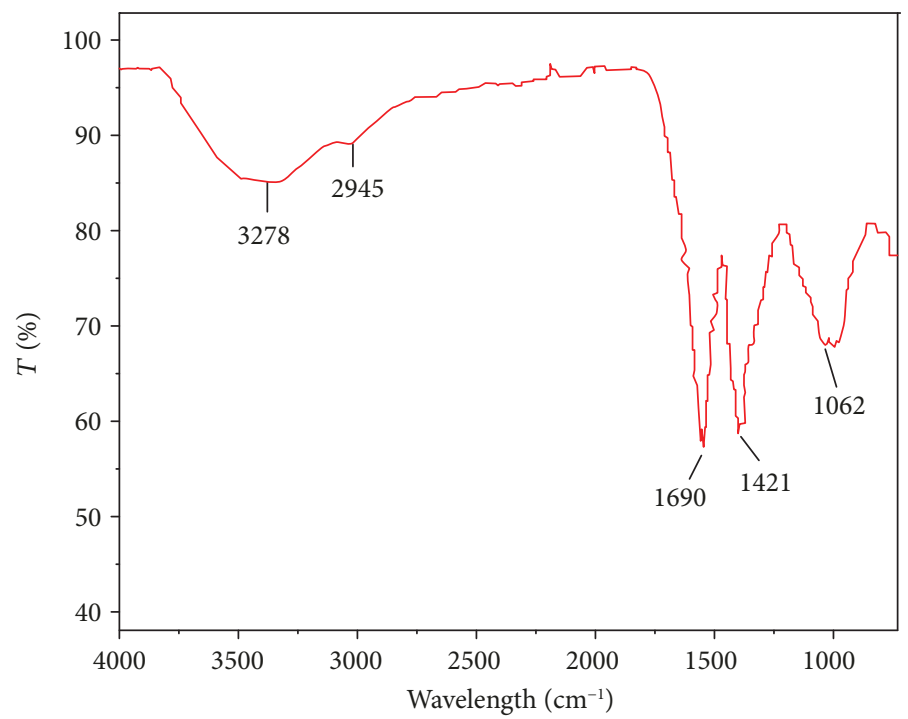

(b)

FIGURE 7: Infrared spectrum of CQDs of (a) tea and (b) peanut shell as carbon source.

black tea as an experimental sample and divided it into eight grades (Super, First, Second, Third, Fourth, Fifth, Sixth, and Outside). For each grade, three tea samples were taken for experiment. We used SPSS software to perform principal component analysis on the quantum dot fluorescence curve (Figure 8(a)) after black tea annihilation. The cumulative contribution rate of the first principal component and the second principal component is $99.15 \%$, which was sufficient to indicate that the two data could sufficiently represent the information of the sample. We constructed the twodimensional image by taking the first principal component and the second principal component data as the $x$-axis and the $y$-axis, respectively. It could be seen from Figure 8(b) that each grade of tea sample had a certain regional distribution. This provided an idea for using quantum dots to discriminate tea grades or food quality in the future.

\section{Conclusions}

We used waste tea leaves and peanut shells as carbon sources to synthesize CQDs. This method has advantages of extremely low cost and easy manipulation. The synthesized light yellow CQD solution emitted strong blue fluorescence under the irradiation of ultraviolet light with a wavelength of $365 \mathrm{~nm}$. After the optimization of preparing conditions and characterization of CQDs, we found the best preparation condition is $200^{\circ} \mathrm{C}$. It is known that the CQDs are $7 \sim 9 \mathrm{~nm}$ in size, spherical in shape, and homogenous in water. The surface contains a large number of hydrophilic groups. Furthermore, we used CQDs to discriminate tea grades with high accuracy. The results obtained in this work provided a way to reduce costs for the practical application of carbon quantum dots in the future. 


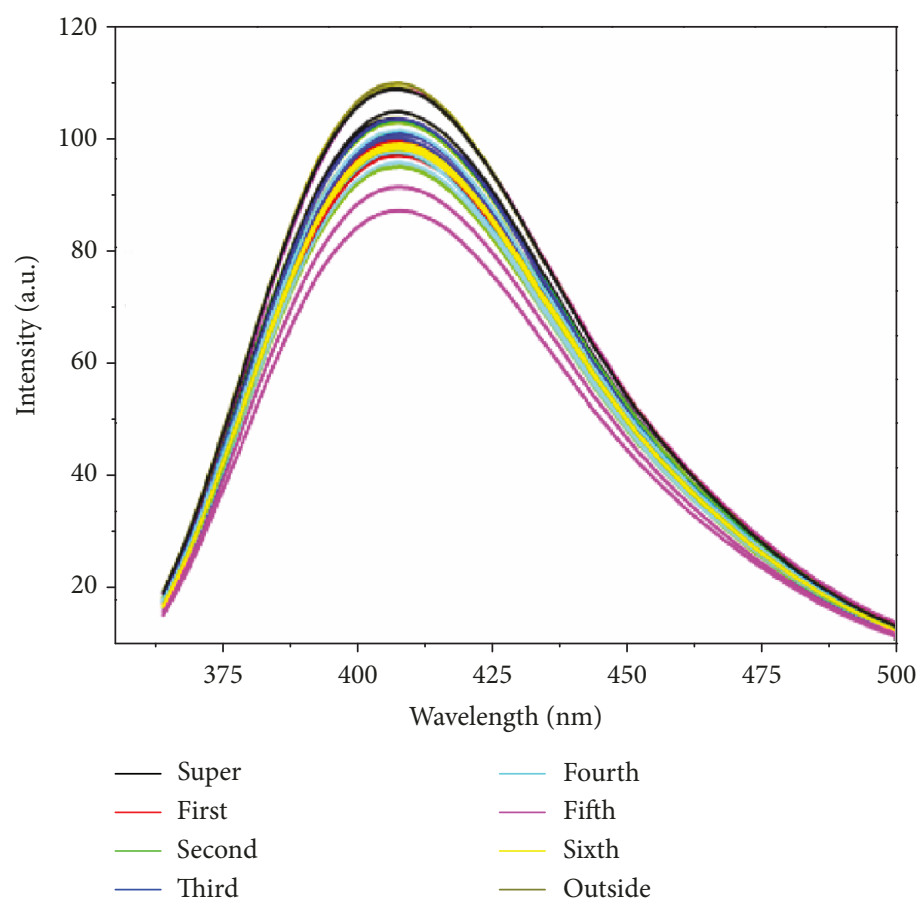

(a)

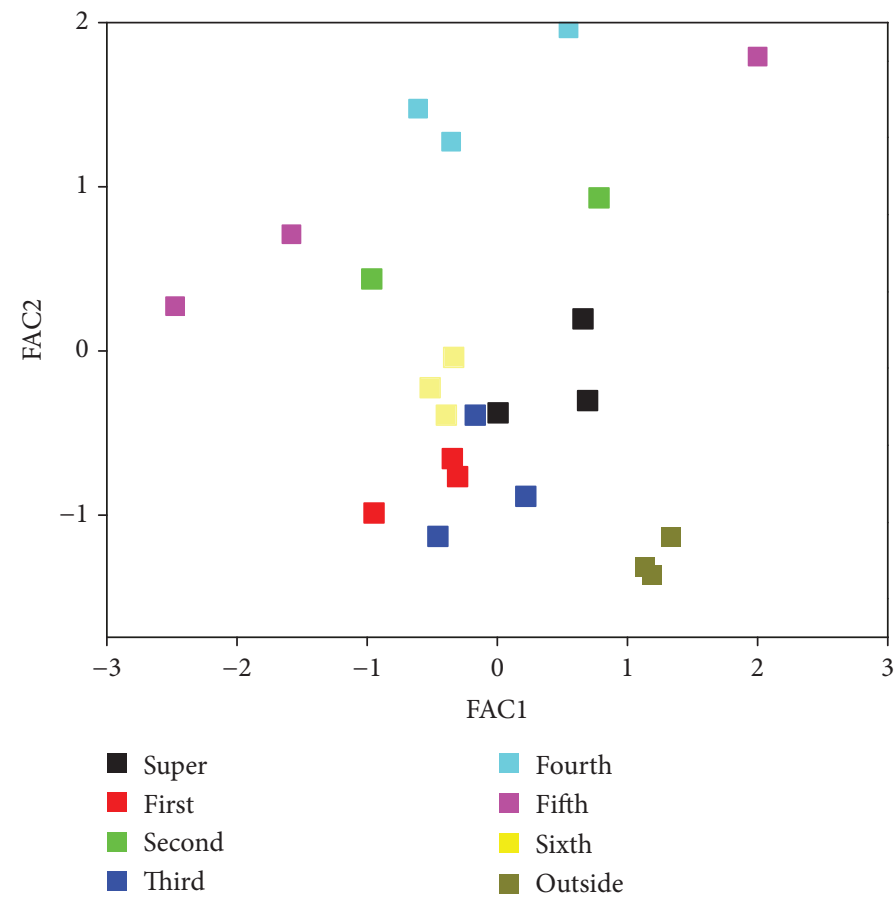

(b)

FIgURE 8: Tea grades of (a) fluorescence spectra and (b) distribution map based on principal component.

\section{Data Availability}

The data used to support the findings of this study are available from the corresponding authors upon request.

\section{Conflicts of Interest}

The authors declare no conflict of interest.

\section{Authors' Contributions}

Jing Zhu and Fengyuan Zhu contributed equally to this work.

\section{Acknowledgments}

This study was financially supported by the National Natural Science Foundation of China (NSFC, 51702004), the 
Scientific Research Foundation of Anhui Agricultural University (Grant No. yj2017-06), the Youth Research Foundation of Anhui Agricultural University (2016ZR003), the Natural Science Foundation of Anhui Province (1808085QE158), and the Open Fund of State Key Laboratory of Tea Plant Biology and Utilization (SKLTOF20180107).

\section{References}

[1] K. M. Tripathi, A. Sachan, M. Castro, V. Choudhary, S. K. Sonkar, and J. F. Feller, "Green carbon nanostructured quantum resistive sensors to detect volatile biomarkers," Sustainable Materials and Technologies, vol. 16, pp. 1-11, 2018.

[2] S. C. Ray, A. Saha, N. R. Jana, and R. Sarkar, "Fluorescent carbon nanoparticles: synthesis, characterization, and bioimaging application," The Journal of Physical Chemistry C, vol. 113, no. 43, pp. 18546-18551, 2009.

[3] F. Zhu, J. Zhu, and Z. Zhang, "Selective detection of glufosinate using $\mathrm{CuInS}_{2}$ quantum dots as a fluorescence probe," RSC Advances, vol. 7, no. 76, pp. 48077-48082, 2017.

[4] Y. Yang, J. Cui, M. Zheng et al., "One-step synthesis of aminofunctionalized fluorescent carbon nanoparticles by hydrothermal carbonization of chitosan," Chemical Communications, vol. 48, no. 3, pp. 380-382, 2012.

[5] F. Yan, D. Kong, Y. Luo et al., "Carbon dots serve as an effective probe for the quantitative determination and for intracellular imaging of mercury(II)," Microchimica Acta, vol. 183, no. 5, pp. 1611-1618, 2016.

[6] A. Cayuela, C. Carrillo-Carrión, M. L. Soriano, W. J. Parak, and M. Valcárcel, "One-step synthesis and characterization of N-doped carbon nanodots for sensing in organic media," Analytical Chemistry, vol. 88, no. 6, pp. 3178-3185, 2016.

[7] S. Sahu, B. Behera, T. K. Maiti, and S. Mohapatra, "Simple onestep synthesis of highly luminescent carbon dots from orange juice: application as excellent bio-imaging agents," Chemical Communications, vol. 48, no. 70, pp. 8835-8837, 2012.

[8] T. Lai, E. Zheng, L. Chen et al., "Hybrid carbon source for producing nitrogen-doped polymer nanodots: one-pot hydrothermal synthesis, fluorescence enhancement and highly selective detection of Fe(iii)," Nanoscale, vol. 5, no. 17, pp. 8015-8021, 2013.

[9] S. Zhu, Y. Song, X. Zhao, J. Shao, J. Zhang, and B. Yang, “The photoluminescence mechanism in carbon dots (graphene quantum dots, carbon nanodots, and polymer dots): current state and future perspective," Nano Research, vol. 8, no. 2, pp. 355-381, 2015.

[10] S. Panda, A. Jadav, N. Panda, and S. Mohapatra, "A novel carbon quantum dot-based fluorescent nanosensor for selective detection of flumioxazin in real samples," New Journal of Chemistry, vol. 42, no. 3, pp. 2074-2080, 2018.

[11] Y. Wang and A. Hu, "Carbon quantum dots: synthesis, properties and applications," Journal of Materials Chemistry C, vol. 2, no. 34, pp. 6921-6939, 2014.

[12] A. Tyagi, K. M. Tripathi, N. Singh, S. Choudhary, and R. K. Gupta, "Green synthesis of carbon quantum dots from lemon peel waste: applications in sensing and photocatalysis," RSC Advances, vol. 6, no. 76, pp. 72423-72432, 2016.

[13] L. Wang, J. Hou, H. Li et al., "Facile synthesis of nitrogendoped carbon dots and its application as sensing probes for serum iron," Journal of Nanoparticle Research, vol. 17, no. 11, p. $457,2015$.
[14] S. Y. Lim, W. Shen, and Z. Gao, "Carbon quantum dots and their applications," Chemical Society Reviews, vol. 44, no. 1, pp. 362-381, 2015.

[15] Y. Zhang, L. Hu, Y. Sun et al., "One-step synthesis of chiral carbon quantum dots and their enantioselective recognition," RSC Advances, vol. 6, no. 65, pp. 59956-59960, 2016.

[16] X. Gao, C. Du, Z. Zhuang, and W. Chen, "Carbon quantum dot-based nanoprobes for metal ion detection," Journal of Materials Chemistry C, vol. 4, no. 29, pp. 6927-6945, 2016.

[17] P. G. Luo, F. Yang, S.-T. Yang et al., "Carbon-based quantum dots for fluorescence imaging of cells and tissues," RSC Advances, vol. 4, no. 21, pp. 10791-10807, 2014. 


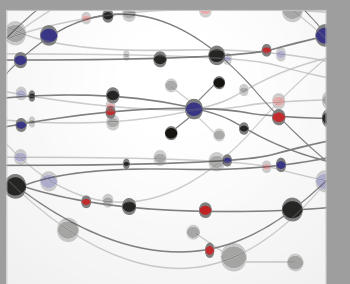

The Scientific World Journal
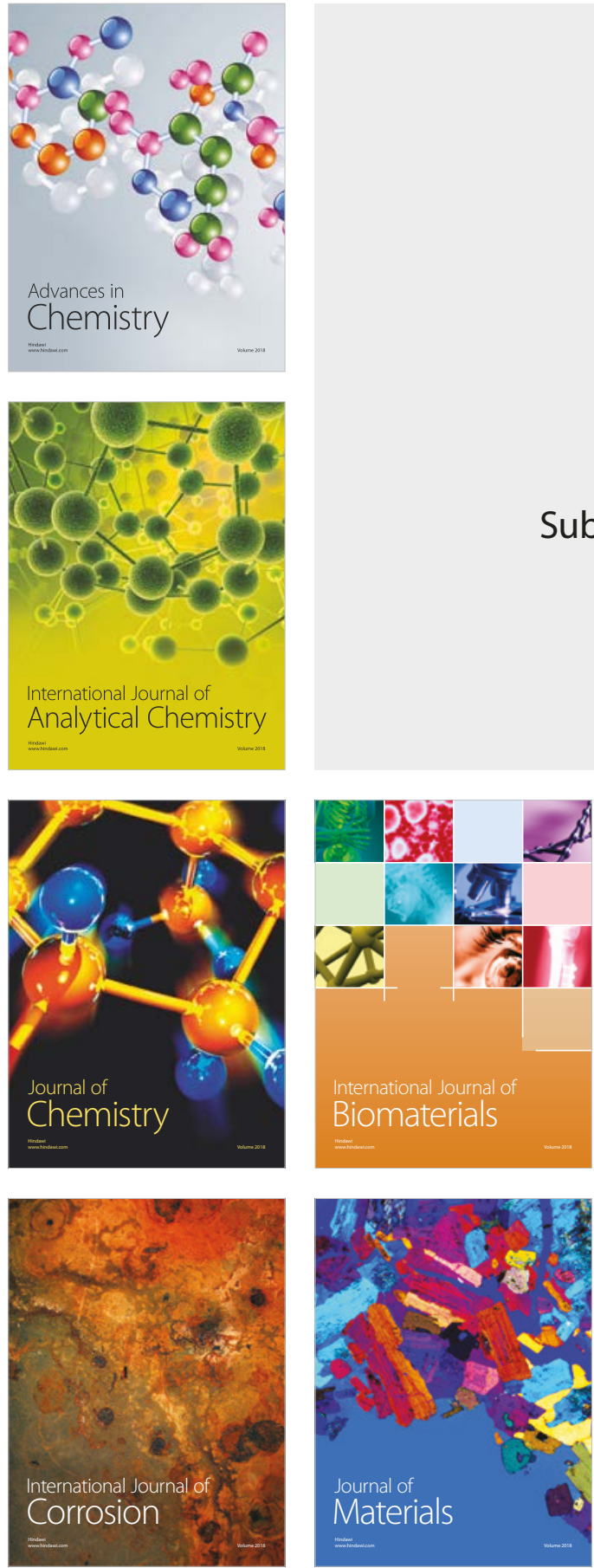

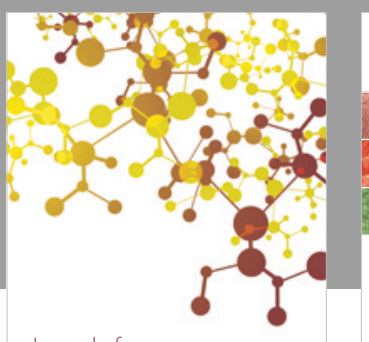

Journal of

Applied Chemistry
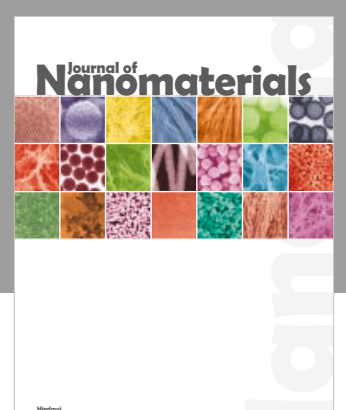

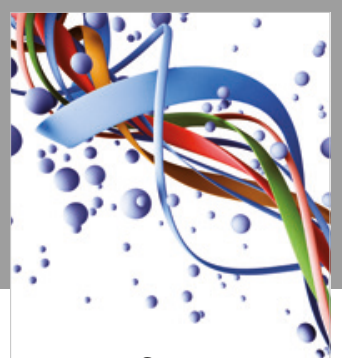

Scientifica

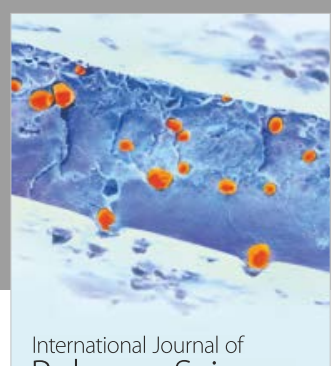

Polymer Science

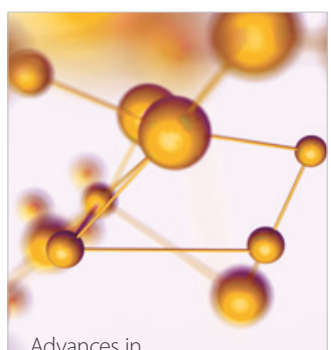

Physical Chemistry
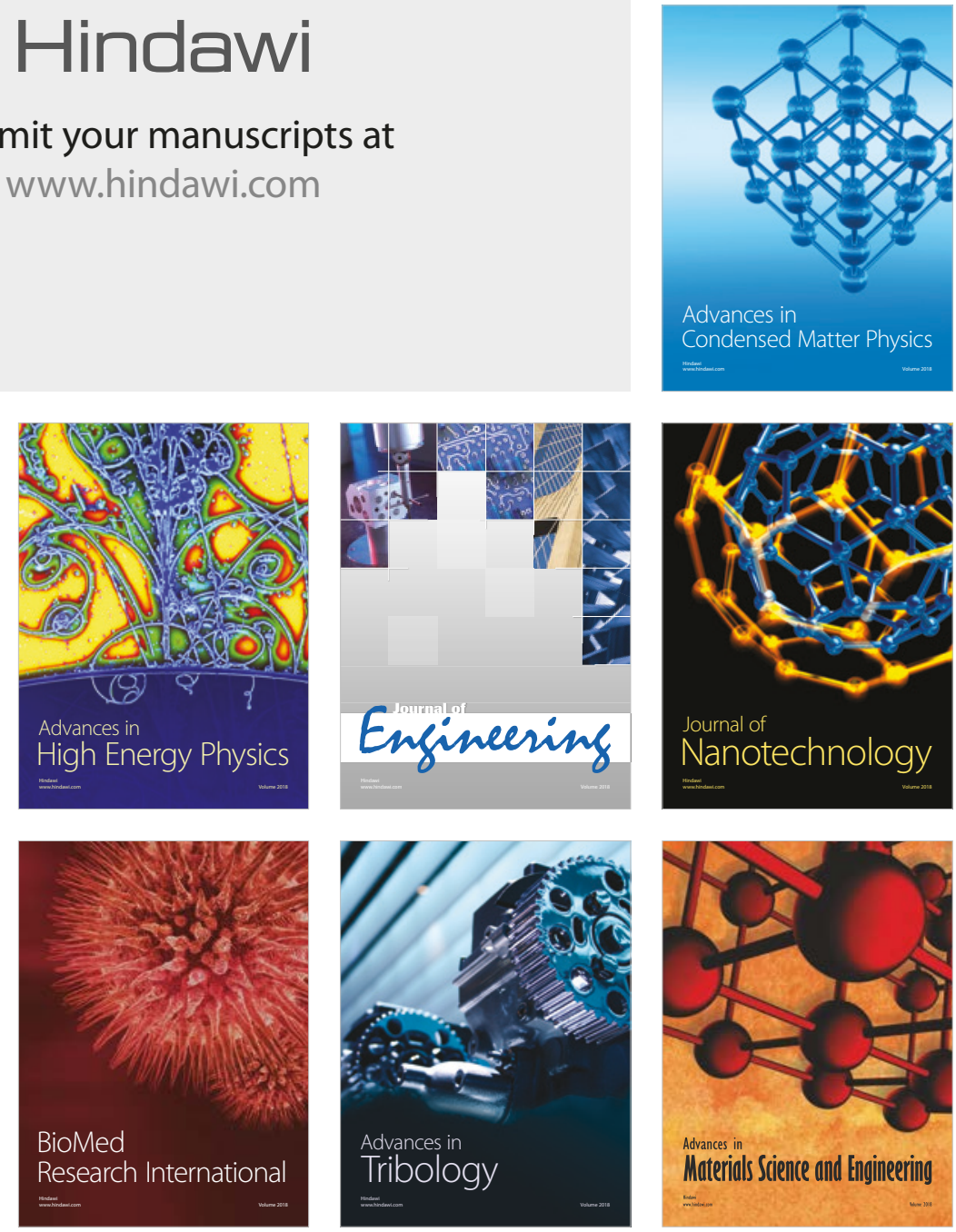